\title{
Evaluation of the neutron background in CsI target for WIMP direct detection when using a reactor neutrino detector as a neutron veto system
}

\author{
Ye Xu ${ }^{1, a}$, Xiangpan $\mathrm{Ji}^{2}$, Haolin $\mathrm{Li}^{2}$, Yulong Feng ${ }^{2}$ \\ ${ }^{1}$ Department of Mathematics and Physics, Fujian University of Technology, Fuzhou 350118, China \\ ${ }^{2}$ School of Physics, Nankai University, Tianjin 300071, China
}

Received: 15 December 2013 / Accepted: 23 March 2014 / Published online: 5 April 2014

(C) The Author(s) 2014. This article is published with open access at Springerlink.com

\begin{abstract}
A direct Weakly Interacting Massive Particle (WIMP) detector with a neutron veto system is designed to better reject neutrons. An experimental configuration is studied in the present paper: a WIMP detectors with $\mathrm{CsI}(\mathrm{Na})$ target is placed inside a reactor neutrino detector. The neutrino detector is used as a neutron veto device. The neutron background for the experimental design has been estimated using the Geant 4 simulation. The results show that the neutron background can decrease to $\mathrm{O}(0.01)$ events per year per tonne of $\mathrm{CsI}(\mathrm{Na})$. We calculate the sensitivity to spin-independent WIMP-nucleon elastic scattering. An exposure of 1 tonne $x$ year could reach a cross-section of about $3 \times 10^{-11} \mathrm{pb}$.
\end{abstract}

\section{Introduction}

It is indicated by seven year Wilkinson Microwave Anisotropy Probe data combined with measurements of baryon acoustic oscillations and Hubble constant that $\sim 83 \%$ of the matter content in the Universe is non-baryonic dark matter [1-3]. Weakly Interacting Massive Particles (WIMPs) [4], predicted by extensions of the standard model of particle physics, are a well-motivated class of candidates for dark matter. They are distributed in the halo surrounding the Milky Way. WIMPs may be directly detected through measuring nuclear recoils in terrestrial detectors produced by their scattering off target nuclei [5-7]. The nuclear reoils is expected to have a roughly exponential energy distribution with a mean energy in a few tens of $\mathrm{keV}[6,8,9]$.

In direct searches for WIMPs, there are three different methods used to detect the nuclear recoils, including collecting ionization, scintillation and heat signatures induced by them. The background of this detection is made up of electron recoils produced by $\gamma$ and $\beta$ scattering off elec-

a e-mail: xuy @ fjut.edu.cn trons, and nuclear recoils produced by neutrons scattering elastically off target nucleus. It is very efficient discriminating nuclear recoils from electron recoils with pulse shape discrimination, hybrid measurements and so on. The rejection powers of these techniques can even reach $10^{6}[10,11]$. For example, the CDMS-II [10] and EDELWEISS-II [12] experiments measure both ionization and heat signatures using cryogenic germanium detectors in order to discriminate between nuclear and electron recoils, and the XENON100 [13] and ZEPLIN-III [14] experiments measure both ionization and scintillation signatures using two-phase xenon detectors. However, it is very difficult to discriminate between nuclear recoils induced by WIMPs and by neutrons. In some dark matter experiments and researches, tagging neutron is applied to reject neutron background. In the ZEPLIN-III experiment, the $0.5 \%$ Gadolinium (Gd) doped polypropylene is used as the neutron veto device, and its maximum tagging efficiency for neutrons reaches about $80 \%$ [15]. In Ref. [16], the $2 \%$ Gd-doped water is used as the neutron veto, and its neutron background can be reduced to 2.2 (1) events per year per tonne of liquid xenon (liquid argon). In our past work [17], the reactor neutrino detector with $1 \%$ Gd-doped liquid scintillator (Gd-LS) is used as the neutron veto system, and its neutron background can be reduced to about 0.3 per year per tonne of liquid xenon.

Cesium iodide (CsI) crystals as a kind of dark matter target have been applied to dark matter experiments, such as the KIMS experiment [18]. In a dark matter experiment with CsI target, CsI crystals needn't to be cooled with the liquid nitrogen or xenon, so this detector can be of the simpler structure. In the Sun, Lu et al.'s work [19], the rejection power against electron recoil can reach $\mathrm{O}\left(10^{7}\right)$ with Na-doped CsI crystals. The feasibility of direct WIMPs detection with a neutron veto based on a neutrino detector had been validated in our past work [17]. So, in the present paper, a neutrino detector 
with Gd-LS (1 \% Gd-doped) is still used as a neutron-tagged device and WIMP detectors with CsI target are placed inside the Gd-LS. Here we designed an experimental configuration: four WIMP detectors with $\mathrm{CsI}(\mathrm{Na})$ target are individually placed inside four reactor neutrino detector modules which are used as a neutron veto system. The experimental hall of the configuration is assumed to be located in an underground laboratory with a depth of 910 meter water equivalent (m.w.e.), which is similar to the far hall in the Daya Bay reactor neutrino experiment [20]. Collecting scintillation signals is considered as the only method of WIMPs detection in our work. The neutron background for this design are estimated using the Geant4 [21] simulation.

\section{Detector description}

Four identical WIMP detectors with $\mathrm{CsI}(\mathrm{Na})$ target are individually placed inside four identical neutrino detector modules. The concentration of $\mathrm{Na}$ is $0.02 \%$ in CsI crystals. The experimental hall of this experimental configuration is assumed to be located in an underground laboratory with a depth of 910 m.w.e., which is similar to the far hall in the Daya Bay reactor neutrino experiment. The detector is located in a cavern of $20 \times 20 \times 20 \mathrm{~m}^{3}$. The four identical cylindrical neutrino modules (each $413.6 \mathrm{~cm}$ high and $393.6 \mathrm{~cm}$ in diameter) are immersed into a $13 \times 13 \times 8 \mathrm{~m}^{3}$ water pool at a depth of 2.5 meters from the top of the pool and at a distance of $2.5 \mathrm{~m}$ from each vertical surface of the pool. The detector configuration is shown in Fig. 1.

Fig. 1 Cross-section for WIMP detector with CsI targets (a). Longitudinal section for WIMP detector with CsI targets (b). Longitudinal section for a neutrino detector where a WIMP detector is placed inside (c). Four WIMP detectors are individually placed inside four neutrino detectors in a water shield (d)

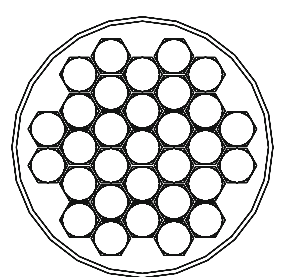

(a)

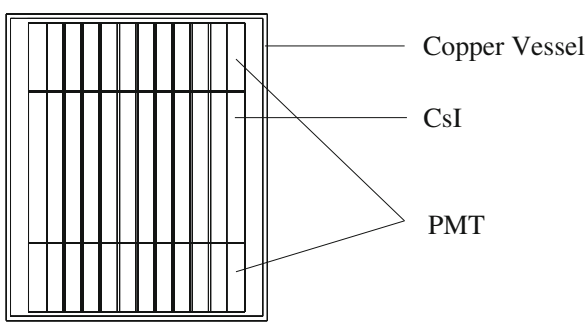

(b)

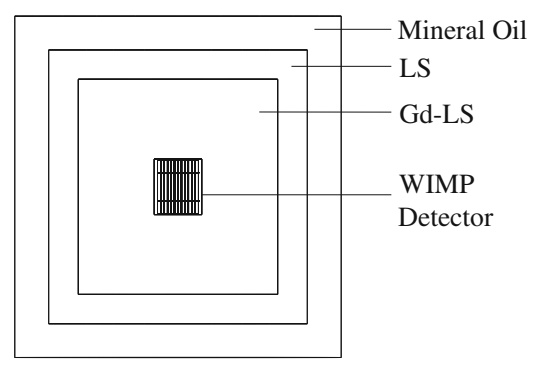

(c)
Each neutrino detector module is partitioned into three enclosed zones. The innermost zone is filled with Gd-LS, which is surrounded by a zone filled with unload liquid scintillator (LS). The outermost zone is filled with transparent mineral oil. 366 8-in. PMTs are mounted in the mineral oil.

Each WIMP detector $(69.6 \mathrm{~cm}$ height, $59.9 \mathrm{~cm}$ in diameter) consists of three components: a $\mathrm{CsI}(\mathrm{Na})$ crystal array, PMTs and Copper vessels. The $\operatorname{CsI}(\mathrm{Na})$ array is made up of $31 \mathrm{CsI}(\mathrm{Na})$ crystals whose sections are regular hexagons (34 cm height, $4.5 \mathrm{~cm}$ side length) and is placed inside a $1 \mathrm{~cm}$ thick copper vessel (69.2 cm height, $59.5 \mathrm{~cm}$ in diameter) filled with dry nitrogen gas. Figure 1 shows the arrangement of these crystals. 62 3-in. PMTs are individually mounted on two ends of these crystals. The copper vessel of each WIMP detector is surrounded by an $0.2 \mathrm{~cm}$ thick aluminum reflector for photons produced in the Gd-LS.

\section{Some features of simulation}

The Geant4 (version 8.2) package [21] has been used in our simulations. The physics list in the simulations includes transportation processes, decay processes, low energy processes, electromagnetic interactions (multiple scattering processes, ionization processes, scintillation processes, optical processes, cherenkov processes, Bremsstrahlung processes, etc.) and hadronic interactions (lepton nuclear processes, fission processes, elastic scattering processes, inelastic scattering processes, capture processes, etc.). The hadronic processes include the low energy $(<20 \mathrm{GeV})$, high energy 
Table 1 Estimation of neutron background from different sources for an underground laboratory at a depth of 910 m.w.e. The column labeled "20 keV $<E_{\text {recoil }}<100 \mathrm{keV"} \mathrm{identifies} \mathrm{the} \mathrm{number} \mathrm{of} \mathrm{neutrons} \mathrm{whose} \mathrm{energy} \mathrm{deposition} \mathrm{in} \mathrm{the} \mathrm{CsI(Na)} \mathrm{target} \mathrm{is} \mathrm{in} \mathrm{the} \mathrm{same} \mathrm{range} \mathrm{as} \mathrm{WIMP}$ interactions. The column labeled "Not Tagged" identifies the number of neutrons which are misidentified as WIMP signatures (their energy deposition in the CsI(Na) target is in the same range as WIMP interactions while their recoil energies in the Gd-LS/LS are less than the energy threshold of $1 \mathrm{MeV}$ ). The row labeled "copper vessel" identifies the number of neutrons from the copper vessels. The row labeled "PMTs in copper vessel" identifies the number of neutrons from the PMTs in the copper vessels. The row labeled "cosmic muons" identifies the number of cosmogenic neutrons in the case of not using the muon veto system. The row labeled "muon veto" identifies the number of cosmogenic neutrons in the case of using the muon veto system. We assume that neutron contamination level from cosmic muons decreases by a factor of 30 using a muon veto system. Only the total background in the case of using the muon veto system is listed in this table. The terms after \pm are errors

\begin{tabular}{lcr}
\hline & $20 \mathrm{keV}<E_{\text {recoil }}<100 \mathrm{keV}$ & Not tagged \\
\hline PMTs in copper vessels & $2.9 \pm 0.46$ & $0.04 \pm 0.006$ \\
Copper vessels & $0.03 \pm 0.005$ & $0.001 \pm 0.0002$ \\
Cosmic muons & $2.5 \pm 0.45$ & $0.28 \pm 0.15$ \\
Muon veto & $0.08 \pm 0.08$ & $0.01 \pm 0.028$ \\
Total (muon veto) & $3.0 \pm 0.47$ & $0.05 \pm 0.03$ \\
\hline
\end{tabular}

$(>20 \mathrm{GeV})$ and neutron high-precision $(<20 \mathrm{MeV})$ models. The cuts for the productions of gammas, electrons and positrons are $1 \mathrm{~mm}, 100 \mu \mathrm{m}$ and $100 \mu \mathrm{m}$, respectively. The quenching factor is defined as the ratio of the detector response to nuclear and electron recoils. The Birks factor for protons in the Gd-LS is set to $0.01 \mathrm{~g} / \mathrm{cm}^{2} / \mathrm{MeV}$, corresponding to the quenching factor 0.17 at $1 \mathrm{MeV}$, in our simulation. Besides, We utilize 10 Intel Core i5 CPUs (four cores, each core offer a base of speed of $2.8 \mathrm{GHz}$ ) in the neutron background evaluation.

\section{Neutron background estimation}

The recoil energies for WIMP interactions with $\mathrm{CsI}(\mathrm{Na})$ nuclei were set to a range from 20 to $100 \mathrm{keV}$ in this work. Proton recoils induced by neutrons and neutron-captured signals are used to tag neutrons which reach the Gd-LS. The energy deposition produced by proton recoils is close to a uniform distribution. Neutrons captured on $\mathrm{Gd}$ and $\mathrm{H}$ lead to a release of about 8 and $2.2 \mathrm{MeV}$ of $\gamma$ particles, respectively. Due to the instrumental limitations of the Gd-LS, we assume neutrons will be tagged if their energy deposition in the Gd-LS is more than $1 \mathrm{MeV}$, corresponding to $0.17 \mathrm{MeVee}$ (electron equivalent energy). In the Gd-LS, it is difficult to distinguish signals induced by neutrons from electron recoils, which are caused by the radioactivities in the detector components and the surrounding rocks. But these radioactivities can be controlled to less than $\sim 50 \mathrm{~Hz}$ according to the Daya Bay experiment [20]. If we assume a $100 \mu$ s for neutron tagging time window, the indistinguishable signals due to the radioactivities will result in a total dead time of less than 44 hours per year.

Neutrons are produced from the detector components and their surrounding rock. For the neutrons from the surrounding rock there are two origins: first by spontaneous fission and $(\alpha, \mathrm{n})$ reactions due to ${ }^{238} \mathrm{U}$ and ${ }^{232} \mathrm{Th}$ in the rock (these neutrons can be omitted because they are efficiently shielded, see Sect. 4.2), and secondly by cosmic muon interactions with the surrounding rock.

We estimated the number of neutron background in the $\mathrm{CsI}(\mathrm{Na})$ target of one tonne. This number has been normalized to one year of data taking and are summarized in Table 1 .

\subsection{Neutron background from detector components}

Neutrons from the detector components are induced by $(\alpha$, n) reactions due to $\mathrm{U}$ and Th. According to Mei et al. [22], the differential spectra of neutron yield can be expressed as

$Y_{i}\left(E_{n}\right)=N_{i} \sum_{j} \frac{R_{\alpha}\left(E_{j}\right)}{S_{i}^{m}\left(E_{j}\right)} \int_{0}^{E_{j}} \frac{d \sigma\left(E_{\alpha}, E_{n}\right)}{d E_{\alpha}} d E_{\alpha}$

where $N_{i}$ is the total number of atoms for the $i$ th element in the host material, $R_{\alpha}\left(E_{j}\right)$ refers to the $\alpha$-particle production rate for the decay with the energy $E_{j}$ from ${ }^{232} \mathrm{Th}$ or ${ }^{238} \mathrm{U}$ decay chain, $E_{\alpha}$ refers to the $\alpha$ energy, $E_{n}$ refers to the neutron energy, and $S_{i}^{m}$ is the mass stopping power of the $i$ th element.

\subsubsection{Neutrons from PMTs in copper vessels}

The $\mathrm{U}$ and Th contaminations in the $\mathrm{SiO}_{2}$ material are considered as the only neutron source in the PMTs in our work. Neutrons from $\mathrm{SiO}_{2}$ are emitted with their average energy of $2.68 \mathrm{MeV}$ [22]. The PMTs in the copper vessels of the four WIMP detectors amounts to 248 . The U and Th concentrations in the PMT components can reach ten or even less ppb [23], so a rate of one neutron emitted per PMT per year is conservatively estimated [24]. Consequently, there are 248 neutrons produced by all the PMTs in the copper vessels per year. A simulated sample of $2.48 \times 10^{6}$ events is used 
to study this neutron background. These events generated isotropically are uniformly distributed in the $\mathrm{SiO}_{2}$ material of the PMTs.

The simulation result is summarized in Table 1. 2.9 neutron events/(ton $\cdot y r)$ reach the $\mathrm{CsI}(\mathrm{Na})$ target and their energy deposition falls in the same range as that of the WIMP interactions, as seen in Table 1. Because 0.04 of them are not tagged in the Gd-LS, these background events cannot be eliminated. The uncertainties of the neutron background from the PMTs in Table 1 are from the binned neutron spectra in Ref. [22]. But the neutron background errors from the statistical fluctuation (their relative errors are less than $1 \%$ ) are too small to be taken into account.

\subsubsection{Neutrons from copper vessels}

In the copper vessels, neutrons are produced by the $\mathrm{U}$ and Th contaminations and emitted with their average energy of $0.81 \mathrm{MeV}$ [22]. Their total volume is about $7.2 \times 10^{4} \mathrm{~cm}^{3}$. The radioactive impurities Th can be reduced to $2.5 \times 10^{-4} \mathrm{ppb}$ in some copper samples [25]. If we conservatively assume a $0.001 \mathrm{ppb} \mathrm{U} / \mathrm{Th}$ concentrations in the copper material [26], a rate of one neutron emitted per $4 \times 10^{4} \mathrm{~cm}^{3}$ per year is estimated [16]. Consequently, there are 1.8 neutrons produced by the all copper vessels per year. A simulated sample of $1.8 \times 10^{5}$ events is used to study this neutron background. These events generated isotropically are uniformly distributed in the copper material of the copper vessels.

The simulation result is summarized in Table 1. 0.03 neutron events/(toncdotyr) reach the $\mathrm{CsI}(\mathrm{Na})$ target and their energy deposition falls in the same range as that of the WIMP interactions (see Table 1). As 0.001 of them are not tagged in the Gd-LS, these background events cannot be eliminated. The uncertainty of the neutron background from the copper vessels in Table 1 are from the binned neutron spectra in the Ref. [22]. But the neutron background errors from the statistical fluctuation (their relative errors are less than $1 \%$ ) are too small to be taken into account.

\subsubsection{Neutrons from other components}

The $\mathrm{U}$ and Th contaminations in other detector components also contribute to the neutron background in our experiment setup. Neutrons from the Aluminum reflectors are emitted with the average energy of $1.96 \mathrm{MeV}$ [22]. The $\mathrm{U}$ and Th contaminations in the carbon material are considered as the only neutron source in the Gd-LS/LS. Neutrons from the GdLS/LS are emitted with the average energy of 5.23 MeV [22]. The $\mathrm{U}$ and $\mathrm{Th}$ contaminations in the $\mathrm{SiO}_{2}$ material are considered as the only neutron source in the PMTs in the oil. Neutrons from PMTs are emitted with the average energy of $2.68 \mathrm{MeV}$ [22]. The $\mathrm{U}$ and Th contaminations in the iron material are considered as the only neutron source in the stainless steel tanks. Neutrons from the stainless steel tanks are emitted with the average energy of $1.55 \mathrm{MeV}$ [22]. We evaluated the neutron background from the above components using the Geant 4 simulation. All the nuclear recoils in the $\mathrm{CsI}(\mathrm{Na})$ target, which fall in the same range as that of the WIMP interactions, are tagged. The neutron background from these components can be ignored. The simulated samples that amount to 1000 years of data taking are used to evaluate these neutron backgrounds.

\subsection{Neutron background from natural radioactivity in the surrounding rock}

In the surrounding rock, almost all the neutrons due to natural radioactivity are below $10 \mathrm{MeV}[16,27]$. Water can be used for shielding neutrons effectively, especially in the low energy range of less than $10 \mathrm{MeV}$ [28]. The WIMP detectors are surrounded by about 2.5 meters of water and more than 1 meter of Gd-LS/LS, so these shields can reduce the neutron contamination from the radioactivities to a negligible level.

\subsection{Neutron background due to cosmic muons}

Neutrons produced by cosmic muon interactions constitute an important background component for dark matter searches. These neutrons with a hard energy spectrum extending to several $\mathrm{GeV}$ energies, are able to travel far from produced vertices.

The total cosmogenic neutron flux at a depth of 910 m.w.e. is evaluated by a function of the depth for a site with a flat rock overburden [29], and it is $1.31 \times 10^{-7} \mathrm{~cm}^{-2} \mathrm{~s}^{-1}$. The energy spectrum (see Fig. 2) and angular distribution of these neutrons are evaluated at the depth of 910 m.w.e. by the method in $[29,30]$. The neutrons with the specified energy and angular distributions are sampled on the surface of the cavern, and the neutron interactions with the detector are simulated with the Geant 4 package. A simulated sample of $1.25 \times 10^{9}$ events is used to study in this neutron background.

Table 1 shows that 2.5 neutron events/(ton $\cdot y r)$ reach the $\mathrm{CsI}(\mathrm{Na})$ target and their energy deposition falls in the same range as that of the WIMP interactions. 0.28 of them are not tagged by the Gd-LS/LS. Muon veto systems can tag muons very effectively, thereby most cosmogenic neutrons can be rejected. In the Daya Bay experiment, the contamination level can even be reduced by a factor of more than 30 [20]. We assume the neutron contamination level from cosmic muons decreases by a factor of 30 using a muon veto system. This could lead to the decrease of cosmogenic neutron contamination to 0.01 events/(ton·yr). The uncertainties of the cosmogenic neutron background in Table 1 are from the statistical fluctuation. 


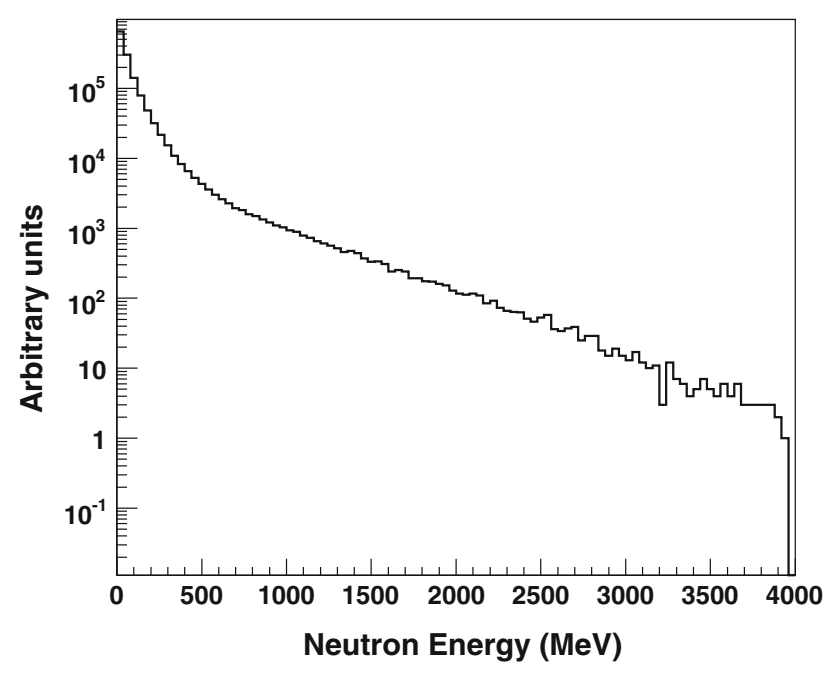

Fig. 2 The energy spectrum of cosmogenic neutrons at depth of 910 m.w.e

\section{Rough estimation of other background}

Besides neutron background, other background events are mainly from reactor neutrino events and electron recoils in the experimental design in the present paper. The contamination caused by electron recoils consists of bulk electron recoil events and surface events.

\subsection{Contamination due to reactor neutrino events}

Since neutrino detectors are fairly close to nuclear reactors (about 2 kilometers away) in reactor neutrino experiments, a large number of reactor neutrinos will pass through the detectors, and nuclear recoils will be produced by neutrino elastic scattering off target nucleus in the WIMP detectors. Although neutrinos may be a source of background for dark matter searches, they can be reduced to a negligible level by setting the recoil energy threshold of $10 \mathrm{keV}$ [31]. Besides, nuclear recoils may also be produced by low energy neutrons produced by the inverse $\beta$-decay reaction $\overline{v_{e}}+p \rightarrow e^{+}+n$. But their kinetic energies are almost below $100 \mathrm{keV}$ [32], and their maximum energy deposition in the WIMP detectors is as large as a few $\mathrm{keV}$. Thus the neutron contamination can be reduced to a negligible level by the energy threshold of 10 $\mathrm{keV}$.

\subsection{Contamination due to bulk electron recoils}

The internal sources of electron recoils are mainly caused by the radioisotopes of ${ }^{137} \mathrm{Cs}$ and ${ }^{87} \mathrm{Rb}$ in the CsI crystals, and the external ones are mainly from the radioisotopes of ${ }^{238} \mathrm{U},{ }^{232} \mathrm{Th}$ and ${ }^{40} \mathrm{~K}$ in the 3 " PMTs inside the copper vessels. Here we assume that the concentrations of the ${ }^{137} \mathrm{Cs}$ and ${ }^{87} \mathrm{Rb}$ in the CsI crystals are about $2 \mathrm{mBq} / \mathrm{kg}$ and $1 \mathrm{ppb}$
[33,34], respectively. Then their background rates will be about 1.7 counts $/ \mathrm{keV} / \mathrm{kg} / \mathrm{day}$ (cpd) in the region $10 \mathrm{keV}$ [34]. While the concentrations of $\mathrm{U} / \mathrm{Th} / \mathrm{K}$ are about 78,25 and $504 \mathrm{mBq} / \mathrm{PMT}$, respectively [34], and their background rates will be about $0.5 \mathrm{cpd}$ in the region $10 \mathrm{keV}$. Considering the fact that the rejection power against electron recoils can reach $\mathrm{O}\left(10^{7}\right)[19]$, we roughly estimate that the bulk electron recoil contamination due to the internal and external sources of the $\mathrm{CsI}(\mathrm{Na})$ crystals is about 0.9 events/(ton $\cdot y r)$.

\subsection{Contamination due to surface events}

The surface events for $\mathrm{CsI}(\mathrm{Na})$ crystals are caused by the deliquescence which reduces the $\mathrm{Na}^{+}$concentration on the crystal surface [19]. Signals produced by $\alpha$ particles from the progenies of ${ }^{222} \mathrm{Rn}$ in the air on the "old" surface of the crystal mimic nuclear recoils seriously. The surface events can be prevented from by avoiding the deliquescence of the crystal surface. For example, the copper vessels are filled with dry nitrogen gas [34], and thus there is no air between the copper vessels and $\mathrm{CsI}(\mathrm{Na})$ crystals. Hence the surface event contamination can be reduced to a negligible level by the above methods.

\section{Discussion and conclusion}

Neutron background can be effectively suppressed neutrino detectors used as a neutron veto system in direct dark matter searches. Table 1 shows the total neutron contamination are 0.05 events/(ton-yr). And compared to Ref. [17], it is reduced by a factor of about 6 . If the electron recoils contaminations are considered, the total background amounts to about 1 events/(ton·yr).

According to our work, the neutron background is mainly from the PMTs in the copper vessels in this configuration with the $\operatorname{CsI}(\mathrm{Na})$ targets. After finishing a precision measurement of the neutrino mixing angle $\theta_{13}$, we can utilize the existing experiment hall and neutrino detectors. This will not only save substantial cost and time for direct dark matter searches, but the neutron background could also decrease to $\mathrm{O}(0.01)$ events per year per tonne of $\mathrm{CsI}(\mathrm{Na})$ in the case of the Daya Bay experiment. According to Ref. [29], The neutron fluxes in the RENO (in an underground laboratory with a depth of 450 m.w.e.), Double CHOOZ (in an underground laboratory with a depth of 300 m.w.e.) experiments $[35,36]$ are respectively about 5 and 3 times more than that of the Daya Bay experiment. Their neutron backgrounds are roughly estimated to be about 0.1 events/(ton-yr), if their detector configurations are the same as the one described above. The neutron contamination is one order of magnitude smaller than the electron recoil contamination, so neutron contamination can be ignored in this detector configuration. 


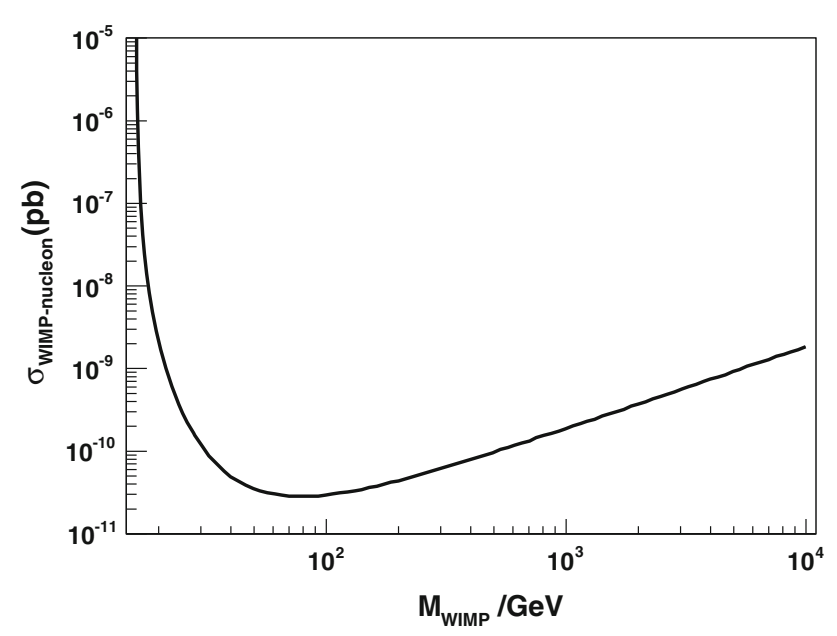

Fig. 3 We calculate the sensitivity to spin-independent WIMP-nucleon elastic scattering assuming an exposure of one tonne $\times$ year. The calculation shows this exposure could reach a cross-section of about $3 \times 10^{-11} \mathrm{pb}$ at the $90 \%$ confidence level. The tool from Ref. [38] has been used

To evaluate the detector capability of directly detecting dark matter, we assume a standard dark matter galactic halo [9], an energy resolution that amounts to $25 \%$ for the energy range of interest and $40 \%$ nuclear recoil acceptance [18].

If no signals are significantly observed, a sensitivity to WIMP-nucleon spin-independent elastic scattering can be calculated via the same method as Ref. [37]. Our calculation shows that an exposure of one tonne $\times$ year could reach a cross-section of about $3 \times 10^{-11} \mathrm{pb}$ at the $90 \%$ confidence level (see Fig. 3).

Acknowledgments This work is supported in part by the National Natural Science Foundation of China (NSFC) under contract No. 11235006 and the science fund of Fujian University of Technology under contract No. GY-Z13114.

Open Access This article is distributed under the terms of the Creative Commons Attribution License which permits any use, distribution, and reproduction in any medium, provided the original author(s) and the source are credited.

Funded by $\mathrm{SCOAP}^{3}$ / License Version CC BY 4.0.

\section{References}

1. N. Jarosik et al., ApJS 192, 14 (2011)

2. W.J. Percival et al., MNRAS 401, 2148 (2010)

3. A.G. Riess et al., ApJ 699, 539 (2009)

4. G. Steigman, M.S. Turner, Nucl. Phys. B 253, 375 (1985)

5. M.W. Goodman, E. Witten, Phys. Rev. D 31, 3059 (1985)

6. G. Jungman, M. Kamionkowski, K. Griest, Phys. Rep. 267, 195 (1996)

7. R.J. Gaitskell, Ann. Rev. Nucl. Part. Sci. 54, 315 (2004)

8. G. Bertone, D. Hooper, J. Silk, Phys. Rept. 405, 279 (2005)

9. J.D. Lewin, P.F. Smith, Astropart. Phys. 6, 87 (1996)

10. Z. Ahmed et al., Science 327, 1619 (2010)

11. W.H. Lippincott et al., Phys. Rev. C 78, 035801 (2008)

12. E. Armengaud et al., Phys. Lett. B 702, 329 (2011)

13. E. Aprile et al., Astropart. Phys. 35, 573 (2012)

14. D. Akimov et al., Phys. Lett. B 709, 14 (2012)

15. D. Akimov et al., Astropart. Phys. 34, 151 (2010)

16. A. Bueno, M.C. Carmona, A.J. Melgarejo, JCAP 08, 019 (2008)

17. Ye Xu, et al., JCAP 06, 009 (2011)

18. S.C. Kim et al., Phys. Rev. Lett. 108, 181301 (2012)

19. X. Sun, L. Junguang et al., Nucl. Instrum. Meth. A 642, 52-58 (2011)

20. F.P. An, Daya Bay Collaboration, et al., Phys. Rev. Lett. 108, 171803 (2012). arXiv:hep-ex/0701029v1

21. S. Agostinelli et al., Nucl. Instrum. Meth. A 506, 250 (2003)

22. D.M. Mei, C. Zhang, A. Hime, Nucl. Instrum. Meth. A 606, 651 (2009)

23. M.J. Carson et al., Astropart. Phys. 21, 667 (2004)

24. A. Bueno, M.C. Carmona, A.J. Melgarejo, JCAP 08, 019 (2008). arXiv:0805.0694

25. Marin E. Keillor et al., J. Radioanal. Nucl. Chem. 282, 703-708 (2009)

26. D.S. Leonard et al., Nucl. Instrum. Meth. A 591, 490 (2008)

27. M.J. Carson et al., Astropart. Phys. 21, 667 (2004)

28. J.M. Carmona et al., Astropart. Phys. 21, 523 (2004)

29. D.M. Mei, A. Hime, Phys. Rev. D 73, 053004 (2006)

30. Y.F. Wang et al., Phys. Rev. D 64, 013012 (2001)

31. J. Monroe, P. Fisher, Phys. Rev. D 76, 033007 (2007)

32. M. Apollonio, et al., CHOOZ Collaboration, Eur. Phys. J. C 27, 331-374 (2003). arXiv:hep-ex/0301017

33. T.Y. Kim et al., Nucl. Instrum. Meth. A 500, 337-344 (2003)

34. H.S. Lee et al., Nucl. Instrum. Meth. A 571, 644-655 (2007)

35. J.K. Ahn et al., Phys. Rev. Lett. 108, 191802 (2012)

36. F. Ardellier, et al., Double Chooz: a search for the neutrino mixing angle $\theta_{13}$. arXiv:hep-ex/0606025

37. G.J. Feldman, R.D. Cousins, Phys. Rev. D 57, 3873 (1998)

38. http://pisrv0.pit.physik.uni-tuebingen.de/darkmatter/limits/index. php 